\title{
ARTICLE \\ Efficient Feature Selection and ML Algorithm for Accurate Diagnostics
}

\author{
Vincent Omollo Nyangaresi $^{1^{*}}$ Nidhal Kamel Taha El-Omari $^{2}$ Judith Nyakanga Nyakina $^{3}$ \\ 1. Faculty of Biological \& Physical Sciences, Tom Mboya University College, Homabay, Kenya \\ 2. The World Islamic Science and Education University, Amman, Jordan \\ 3. School of Nursing, Uzima University, Kisumu, Kenya
}

\section{ARTICLE INFO}

Article history

Received: 19 October 2021

Accepted: 19 January 2022

Published Online: 25 January 2022

Keywords:

Accuracy

Classifier

Ensemble

F-measure

Machine learning

Precision

Recall

\section{Introduction}

Machine learning (ML) and data mining (DM) algorithms present powerful statistical and probabilistic techniques that permit intelligent systems to learn from previous experiences ${ }^{[1]}$. This learning is critical for detection and identification of patterns in the underlying

\begin{abstract}
Machine learning algorithms have been deployed in numerous optimization, prediction and classification problems. This has endeared them for application in fields such as computer networks and medical diagnosis. Although these machine learning algorithms achieve convincing results in these fields, they face numerous challenges when deployed on imbalanced dataset. Consequently, these algorithms are often biased towards majority class, hence unable to generalize the learning process. In addition, they are unable to effectively deal with high-dimensional datasets. Moreover, the utilization of conventional feature selection techniques from a dataset based on attribute significance render them ineffective for majority of the diagnosis applications. In this paper, feature selection is executed using the more effective Neighbour Components Analysis (NCA). During the classification process, an ensemble classifier comprising of K-Nearest Neighbours (KNN), Naive Bayes (NB), Decision Tree (DT) and Support Vector Machine (SVM) is built, trained and tested. Finally, cross validation is carried out to evaluate the developed ensemble model. The results shows that the proposed classifier has the best performance in terms of precision, recall, F-measure and classification accuracy.
\end{abstract}

dataset. As such, they present vital mechanisms for discovering hidden relationships in sophisticated datasets ${ }^{[2]}$. Basically, ML and DM algorithms endeavour to offer reliability and trustworthiness in predictive models so as to boost precision and accuracy ${ }^{[2]}$. As such, these algorithms have found applications in optimization and prediction problems in communication networks and

\footnotetext{
*Corresponding Author:

Vincent Omollo Nyangaresi,

Faculty of Biological \& Physical Sciences, Tom Mboya University College, Homabay, Kenya;

Email:vnyangaresi@tmuc.ac.ke
} 
medical fields, among others. For instance, fuzzy logic (FL) and adaptive neural networks (ANNs) have been deployed in ${ }^{[3]}$ for optimization while authors in ${ }^{[4]}$ have utilized Multi-Layer Feed Forward Network (MFNN) and FL for prediction. On the other hand, MFNN has been deployed in ${ }^{[5]}$ for target cell prediction, while FL has been utilized in ${ }^{[6]}$ for efficiency enhancement.

An adaptive neuro-fuzzy inference system (ANFIS) has been deployed in ${ }^{[7]}$ for destination network prediction. Similarly, FL has been deployed in ${ }^{[8]}$ for target network prediction, while neuro-fuzzy technique is presented in ${ }^{[9]}$ for delay optimization. FL based estimation technique is presented in ${ }^{[10]}$, while authors in ${ }^{[11]}$ have developed a neural network system for predicting the number of users in a network. To boost quality of service (QoS) in $5 \mathrm{G}$ networks, authors in ${ }^{[12]}$ present ANN-FL scheme. Similarly, ANN algorithm is deployed in ${ }^{[13]}$ for QoS and Quality of Experience (QoE) enhancement. ANFIS scheme is presented in ${ }^{[14]}$ for handover optimization, while multilayer neural network (MLNN) technique is introduced in ${ }^{[15]}$ for QoS and QoE enhancement. Similarly, ML based technique for optimizing QoS is presented in ${ }^{[16]}$. On the other hand, a multilayer perception neural network (MPNN) is presented in ${ }^{[17]}$ for delay reduction. Similarly, a neuro-fuzzy based technique is developed in ${ }^{[18]}$ for QoS optimization.

In the medical field, the application of ML algorithms for prediction and classification is on the increase ${ }^{[1]}$. This is specifically the case for cancer prediction, owing to the multi-facet nature of this disease. Here, ML techniques are critical for the extraction of useful information from heterogeneous, complex and large clinical data ${ }^{[19]}$. As explained in ${ }^{[20]}$, ML techniques are also significant for psychopathology risk algorithms that are critical for preventive intervention. For instance, supervised ML techniques are vital for internalizing disorder (ID) early detection. Although many ML schemes have been developed for medical diagnosis, there is need to optimize and improve these algorithms ${ }^{[21]}$. One such improvement is ensemble learning in which a classifier comprises of a set of individual classifiers that are coupled with techniques such as majority voting ${ }^{[22]}$. Here, the ensemble classifier amalgamates the predictions of individual classifiers, and hence exhibit better performance compared with individual classifiers ${ }^{[23-25]}$. This can be attributed to the utilization of various decision making systems that deploy numerous strategies. Consequently, an ensemble classifier benefits from performance of diverse classifiers as well as the diversity of errors ${ }^{[26]}$.

In this paper, an ensemble ML algorithm is developed for enhanced diagnostics in the medical field. To evaluate the developed ensemble algorithm, it is applied in breast cancer (BC) data. The choice of $\mathrm{BC}$ is informed by the fact that cancer is one of the leading causes of deaths worldwide. According to ${ }^{[1]}, \mathrm{BC}$ is one of the most common types of cancer, whose recurrence prognosis is critical for patient survival rate enhancement. Consequently, there is need for the early stage prediction of BC. Authors in ${ }^{[27]}$ point out that ML algorithms improvement in terms of effectiveness has received much attention. However, many cancer cases are still being diagnosed late ${ }^{[28]}$. As such, the predictive models for cancer diagnosis need to exhibit extremely low error rates for effective early diagnosis and treatment. In this environment, a need arises for the medical dataset to be carefully managed, owing to its complex nature ${ }^{[29]}$. Any form of predication errors will have serious consequences, and hence the need for accuracy enhancement in ML algorithms. The major contributions of this paper include the following:

- Neighbour Components Analysis (NCA) is deployed for feature selection so as to identify and eliminate irrelevant or redundant features.

- Five predictive models are formulated, trained and tested on the Wisconsin Diagnostic Breast Cancer (WDBC) dataset.

- A number of performance metrics are developed and deployed to evaluate the developed predictive models.

- The results show that the ensemble classifier comprising of KNN, SVM, DT and NB exhibits the best performance compared to individual classifiers.

The rest of this paper is organized as follows: Section 2 details related work in machine learning algorithms and diagnosis, while Section 3 outlines the adopted system model. On the other hand, Section 4 presents the results and discussion of these results, while Section 5 concludes the paper and gives future directions.

\section{Related Work}

The deployment of machine learning algorithms for diagnostics has received much attention among medical practitioners. As such, numerous schemes have been presented in literature. For instance, neural network based ensemble classifier has been presented in ${ }^{[30]}$ for heart failure detection, yielding high classification accuracy. On the other hand, authors in ${ }^{[1]}$ have applied Random Forest (RF), Linear regression (LR), Multi-layer Perceptron (MLP) and Decision Trees (DT) for breast cancer prediction, with MLP yielding the best performance. Similarly, ANN, DT, support vector machines (SVMs), Naive Bayes(NB), and K-Nearest neighbor (KNN) have 
been deployed for medical diagnosis ${ }^{[31]}$. Among these, ANN is noted to be the best in capturing correlations among attributes. A nested ensemble learning algorithm is developed in ${ }^{[32]}$ for breast cancer diagnosis, while an ensemble classifier for kidney stone prediction is introduced in ${ }^{[33]}$. Similarly, a tree ensemble model is developed in ${ }^{[34]}$ for colorectal cancer survival prediction.

An ensemble learning scheme is presented in ${ }^{[35]}$ for hepatitis disease diagnosis, with results showing that this classifier performed better than individual ANN, ANFIS, KNN and SVM classifiers. An ensemble deep learning algorithm is developed in ${ }^{[36]}$ for heart disease prediction, while an ensemble classifier comprising of Logistic Regression (LR), RF, Adaboost and KNN is introduced in ${ }^{[37]}$ for diabetic retinopathy dataset classification. The results showed that ensemble ML model performed better than individual ML algorithms. On the other hand, an ensemble learner consisting of multiple deep convolutional neural networks (CNNs) is developed in ${ }^{[38]}$ for pulmonary nodules classification. Similarly, ensemble learning technique is presented in ${ }^{[39]}$ for diabetes diagnosis, while semantic segmentation and ensemble learning have been introduced in ${ }^{[40]}$ for cardiovascular pathology assessment.

Genetic algorithm (GA) and RF have been utilized in ${ }^{[41]}$ for $\mathrm{BC}$ detection, while a deep ensemble learning scheme is developed in ${ }^{[24]}$ for Alzheimer's disease (AD) prediction. Similarly, ensemble ML technique is introduced in ${ }^{[20]}$ for internalizing disorders (ID) prediction, while an ensemble of neural network models is presented in ${ }^{[29]}$ for Rheumatoid arthritis (RA) diagnosis. On the other hand, ensemble classifier is developed in ${ }^{[42]}$ for heart disease prediction, while ensemble neural network models are utilized in ${ }^{[43]}$ for medical captioning. An ensemble of deep learning and evolutionary computation is developed in ${ }^{[4]}$ for coronary artery disease prediction, while an ensemble of Bagged Tree (BT), $\mathrm{RF}$ and AdaBoost is developed in ${ }^{[45]}$ for heart disease prediction. In this classifier, Particle Swarm Optimization (PSO) is deployed for feature subset selection. The results showed that BT and PSO attained the highest accuracy. A hybrid of Fuzzy and KNN is deployed in ${ }^{[46]}$ for heart disease prediction, while CNN model is amalgamated with recurrent neural network (RNN) in ${ }^{[47]}$ for lung cancer prediction. An ensemble of CNN and RNN is developed in ${ }^{[48]}$ for COVID-19 diagnosis, while a classifier combining CNNs and SVM is deployed in ${ }^{[49]}$ for COVID-19 classification. On the other hand deep transfer learning scheme are coupled with three $\mathrm{CNN}$ models in ${ }^{[50]}$ and ${ }^{[51]}$ for COVID-19 diagnosis. Similarly, seven pretrained CNN classifiers have been introduced in ${ }^{[52]}$ for
COVID-19 diagnosis from X-ray samples.

Apart from ensemble classifiers, other ML algorithms have also been deployed for diagnostics. For instance, an improved ML is presented in ${ }^{[53]}$ for heart disease prediction, while SVM has been developed in ${ }^{[54]}$ for heart valve diseases diagnosis. On the other hand, a deep RNN model is presented in ${ }^{[55]}$ for prostate cancer diagnosis, while CNNs have been utilized in ${ }^{[56]}$ and ${ }^{[57]}$ for lung cancer prediction. A deep neural network is introduced in ${ }^{[58]}$ for COVID-19 diagnosis. Similarly, a deep CNN is presented in ${ }^{[59]}$ for COVID-19 diagnosis.

Although conventional ML techniques attain admirable classification accuracies in medical diagnoses, their performance diminishes when presented with imbalanced dataset. This is normally the case in detection of minority category ${ }^{[31]}$. In addition, numerous factors negatively impact the performance of current classification models when applied to real data ${ }^{[29]}$. Such issues include class imbalance of the training dataset, and hence these models are often biased towards majority class. Consequently, they are unable to generalize the learning process. Another challenge facing majority of the ML algorithms is the handling of high-dimensional datasets, owing to lack of a framework that employs diverse data sources ${ }^{[36]}$. In addition, the utilization of conventional feature selection techniques from a dataset based on their significance render them ineffective for disease diagnosis.

Although ensemble learning techniques perform exceptionally better than individual classifiers, optimum selection of diversity classifier members to form an ensemble, and the fusion of individual decisions of the base classifiers into a single decision present some challenges. On the other hand, the current deep learning feature extraction and classification models have limitations in both their feature extraction and weighting approaches. It is evident that major advances have been made in developing deep learning models, which are effective classifiers for detection of high-order data relationships to solve complex tasks. However, these models are curtailed by the fat-short property of transcriptbased data, which negatively impact their cancer diagnosis effectiveness.

\section{System Model}

This section presents the description of the deployed dataset, data pre-processing steps, ensemble classifier model and experimentation.

\subsection{DataSet Description}

The Wisconsin Diagnostic Breast Cancer (WDBC) 
dataset is utilized in this paper. It has 699 instances with 11 features as shown in Table 1.

Table 1. Summary of Attributes

\begin{tabular}{ccc}
\hline Attribute number & Feature name & Domain \\
\hline 1 & Sample code number & $1-683$ \\
2 & Clump Thickness & $1-10$ \\
3 & Uniformity of Cell Size & $1-10$ \\
4 & Uniformity of Cell Shape & $1-10$ \\
5 & Marginal Adhesion & $1-10$ \\
6 & Single Epithelial Cell Size & $1-10$ \\
7 & Bare Nuclei & $1-10$ \\
8 & Bland Chromatin & $1-10$ \\
9 & Normal Nucleoli & $1-10$ \\
10 & Mitoses & $1-10$ \\
$\mathrm{Y}$ & Class & 2 or 4 \\
\hline
\end{tabular}

The class distribution comprises of 241 malignant (representing $34.48 \%$ ) and 458 benign (representing $65.52 \%$ ) subjects. On the other hand, the target label falls into two classes which include benign (2) or malignant (4).

\subsection{Data Pre-processing}

Prior to the classification process, data cleaning was executed to eliminate or lessen noise in the data. During this process, 16 instances for the Bare Nuclei feature were found to be missing and hence were eliminated from the dataset. In addition, the Sample code number and Class features are irrelevant during the classification process and hence are eliminated. Consequently, only 9 features and 683 instances remained, out of which 444 were benign while 239 were malignant. Table 2 gives the basic statistics (mean and standard deviation-std) of the remaining features.
Table 2. Attribute statistics

\begin{tabular}{ccc}
\hline Feature name & Average & Std \\
\hline Clump Thickness & 4.44 & 2.82 \\
Uniformity of Cell Size & 3.15 & 3.07 \\
Uniformity of Cell Shape & 3.22 & 2.99 \\
Marginal Adhesion & 2.83 & 2.86 \\
Single Epithelial Cell Size & 3.23 & 2.22 \\
Bare Nuclei & 3.54 & 3.64 \\
Bland Chromatin & 3.45 & 2.45 \\
Normal Nucleoli & 2.87 & 3.05 \\
Mitoses & 1.60 & 1.73 \\
\hline
\end{tabular}

To ensure that all features in the dataset have equal coefficients, the standard scalar was computed such that each feature has a mean of zero and variance of unity. This was followed by the Min-Max scalar computation that shifted the data in a way that all features have a domain of between zero and unity.

\subsection{Ensemble Classifier Model}

In this paper, an ensemble classifier is constructed, consisting of KNN, SVM, DT and NB. As shown in Figure 1, an ensemble model consists of $K$ single classifiers and each single model has $N$ inputs. As such, the entire model has $K^{*} N$ input features and the output of this ensemble model is computed based on majority voting.

The classifiers of the proposed ensemble are individual KNN, DT, SVM and NB models. As such, four ensemble models can be established for classification, which include KNN ensemble (KNNE), DT ensemble (DTE), SVM ensemble (SVME) and NB ensemble (NBE). Consequently, the proposed ensemble model comprises of KNN, DT, SVM and NB classifiers. The DT model

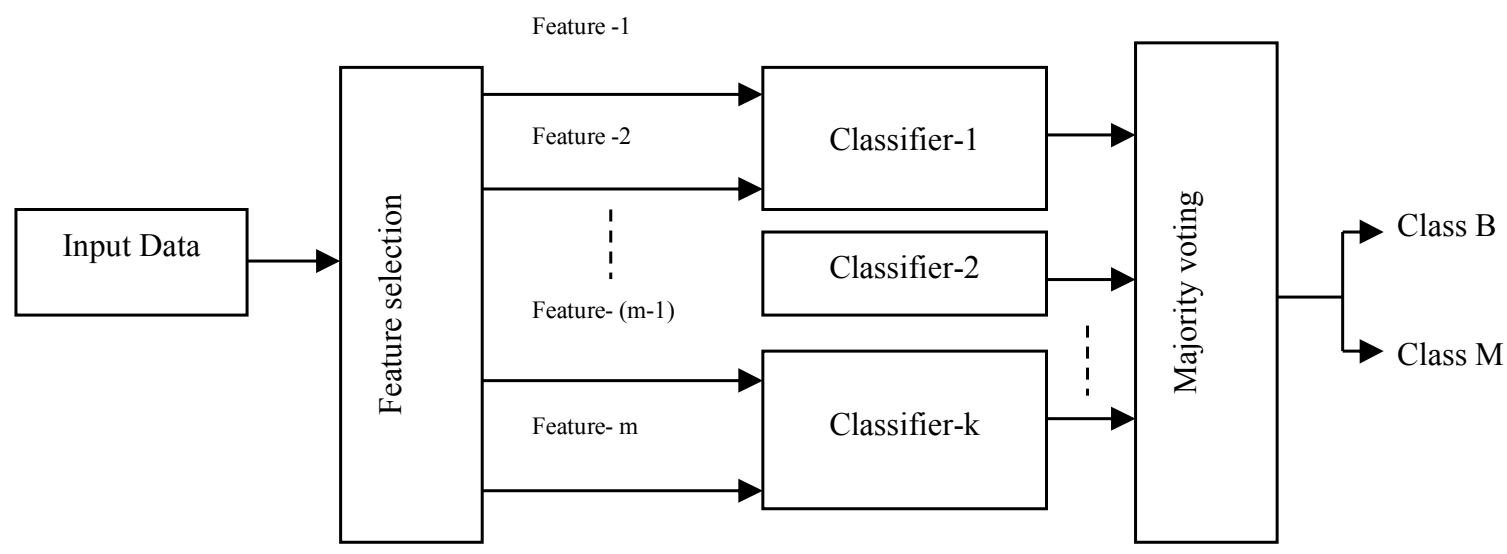

Figure 1. Generalized Ensemble Classifier 
groups data samples based on numerous questions, where the root of the tree comprises of all data samples. Here, the tree is generated through a recursive process as shown in Algorithm 1.

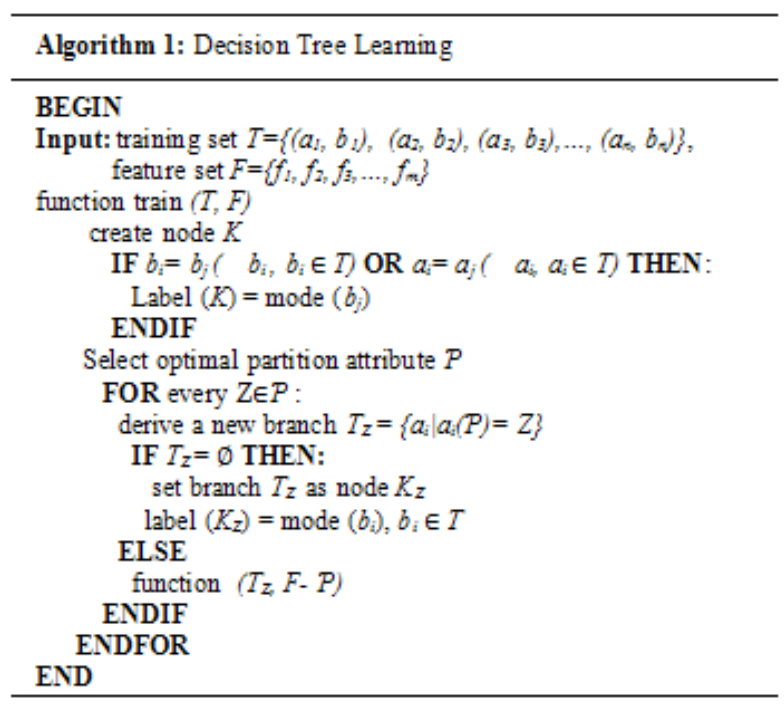

On the other hand, the NB algorithm is a probabilistic classification that derives its probability value by computing the frequency and value combinations from the dataset. The assumption made here is that all features are independent. Algorithm 2 gives the steps followed in this classification.

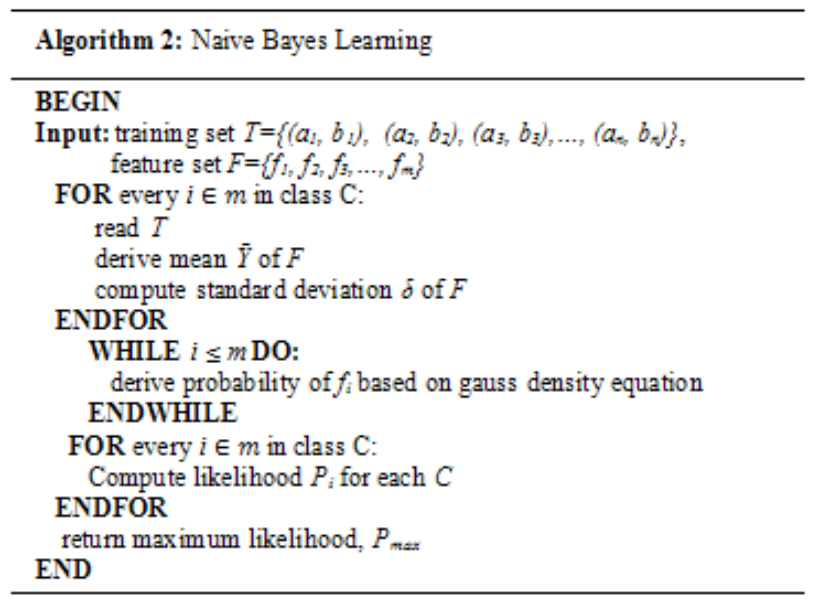

On its part, the KNN is a non-parametric classification algorithm whose input comprises of $k$, which is positive integer representing the number of classes in the underlying dataset. Here, the classification of input data is based on the majority of its neighbours. Consequently, input data is assigned to a class that is higher in its $k$-nearest neighbours as shown in Algorithm 3. The Euclidean distance $D$ is utilized to measure the distance between data points.

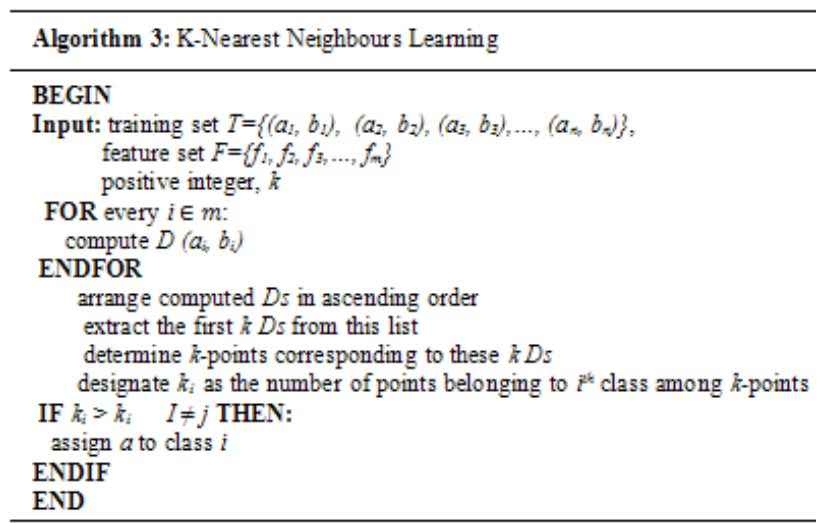

On the other hand, SVM is a classification algorithm that employs subset of training data points in its decision function. These data points become support vectors and this algorithm has been demonstrated to be highly effective for high dimensional data. Algorithm 4 presents the steps necessary for the deployment of SVM.

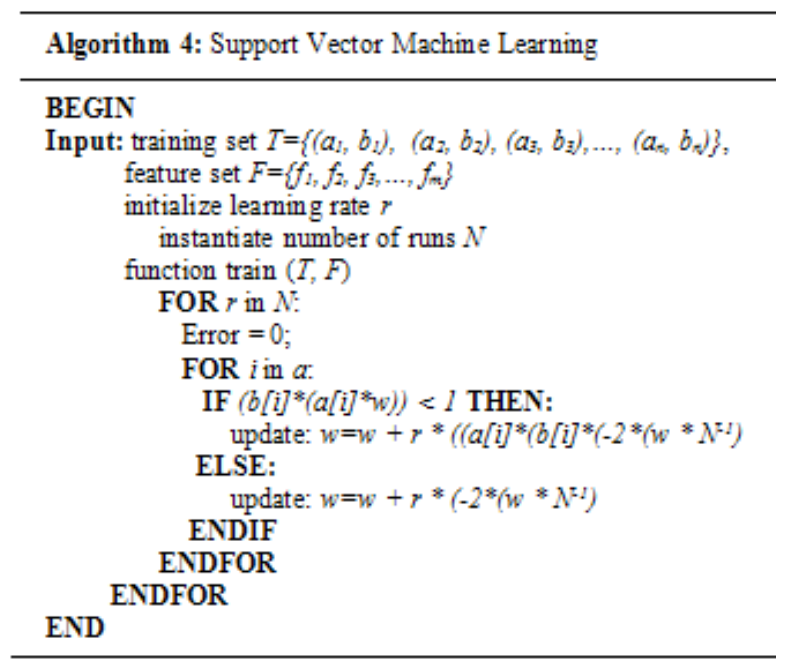

As already alluded, the ensemble model comprises of KNN, DT, SVM and NB whose algorithms have been presented above. The fifth ensemble model consists of some clusters of these classifiers as shown in Figure 2. In this ensemble model, the input features fed to individual classifiers are replicated and similar to each cluster.

In this paper, feature selection is executed using neighbour components analysis (NCA). The aim is to identify and eliminate irrelevant or redundant features so so as to remain with the most relevant ones. NCA was chosen due to its ability to maximize classification accuracy. It does this through dataset dimensionality reduction and hence achieves optimal objective function. Normally, the gradient-based optimizer is deployed for this purpose. Finally, the output of the ensemble model is obtained through majority voting strategy. 


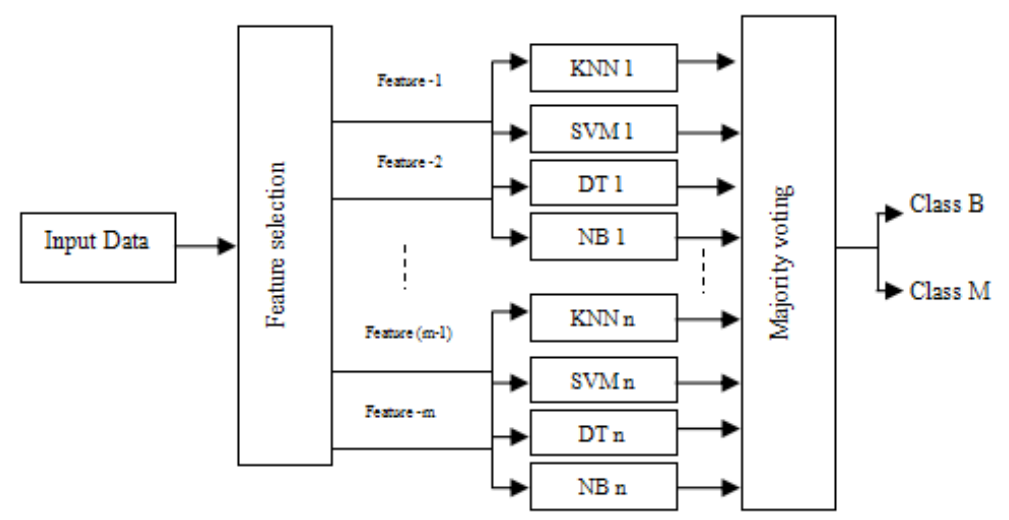

Figure 2. Proposed Ensemble Classifier

\subsection{Experimentations}

During training and testing process, the dataset was portioned into $80 \%$ training instances and $20 \%$ testing instances. The goal is to classify the tumor either as malignant or benign. Feature selection is then accomplished using NCA before individual models are trained, tested and deployed to classify the dataset. This was followed by the deployment of the proposed ensemble model to classify the same dataset as shown in Figure 3.

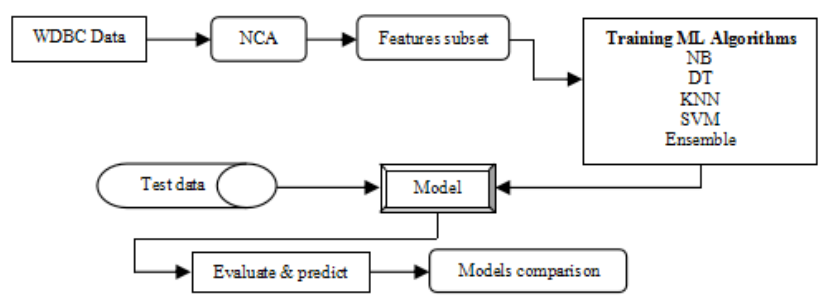

Figure 3. Flowchart of the Proposed Model

Thereafter, comparisons are executed to investigate the effectiveness of the proposed model. To accomplish this, accuracy, precision and recall are deployed as shown in Table 3.

Table 3. Performance Metrics

\begin{tabular}{cc}
\hline Metric & Formulation \\
\hline Accuracy & $\frac{T P+T N}{T P+T N+F P+F N}$ \\
Precision & $\frac{T P}{T P+F P}$ \\
Recall & $\frac{T P}{T P+F N}$ \\
F-measure & $\frac{2 * \text { Precision } * \text { Recall }}{\text { Precision }+ \text { Recall }}$ \\
\hline
\end{tabular}

Here, TP is the true positive, TN is true negative, FP is false positive, and FN is false negative.

\section{Results and Discussion}

In this section, the training and testing results of the developed machine learning algorithms are presented. Table 4 shows the precision, recall and F-measure values obtained for the various classifiers. It is evident that the lowest value for precision was 94.21 which was recorded by NB classifier while the highest value for precision was 99.12 which belonged to the proposed ensemble classifier. Regarding recall, NB had the lowest value of 94.28 while the proposed ensemble classifier had the highest value of 99.45. On the other hand, 94.245 was the lowest F-measure value that was recorded by NB classifier while 99.285 was the highest F-measure value that was recorded by the proposed classifier.

Table 4. Performance Comparisons

\begin{tabular}{cccc}
\hline Classifier & Precision & Recall & F-Measure \\
\hline DT & 95.07 & 95.23 & 95.150 \\
SVM & 98.15 & 98.26 & 98.205 \\
NB & 94.21 & 94.28 & 94.245 \\
KNN & 97.16 & 97.34 & 97.250 \\
Ensemble & 99.12 & 99.45 & 99.285 \\
\hline
\end{tabular}

As shown in Figure 4, the proposed ensemble classifier had the highest values for precision, recall and F-measure. This was followed by SVM, KNN, DT and NB in that order. It is also clear that the values for recall remained the highest among other metrics in all the five classifiers. 


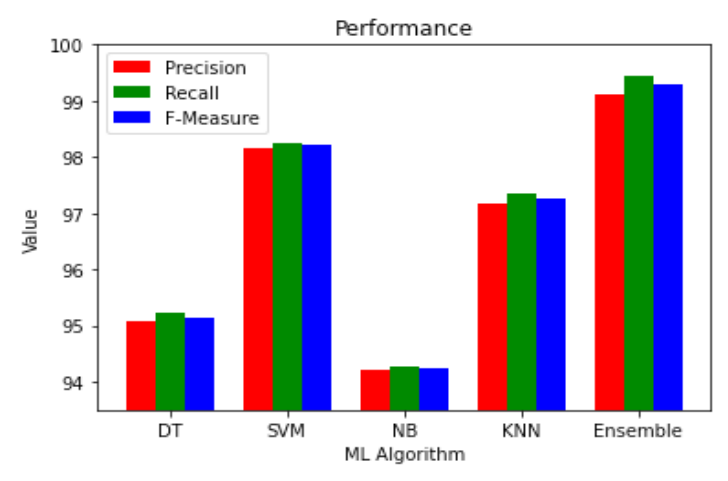

Figure 4. Performance Metrics Comparisons

Table 5 presents the values obtained for prediction accuracy during training and testing. It is clear from Table 5 that during training, the highest value of accuracy was $98.95 \%$ while the lowest value was 94.03 . On the other hand, $99.27 \%$ accuracy was the highest during testing while $94.34 \%$ accuracy was the lowest during testing.

Table 5. Prediction Accuracy

\begin{tabular}{cccc}
\hline \multirow{2}{*}{ Classifier } & \multicolumn{3}{c}{ Prediction Accuracy (\%) } \\
\cline { 2 - 4 } & Training & Testing & Overall \\
\hline DT & 93.65 & 94.91 & 94.280 \\
SVM & 97.98 & 98.56 & 98.270 \\
NB & 94.03 & 94.34 & 94.185 \\
KNN & 97.06 & 97.45 & 97.255 \\
Ensemble & 98.95 & 99.27 & 99.110 \\
\hline
\end{tabular}

Based on Figure 5, NB classifier had the lowest overall classification accuracy of $94.185 \%$ while the proposed ensemble classifier had the highest overall classification accuracy of $99.11 \%$. It is also evident that training had lower classification accuracy compared with testing phase.

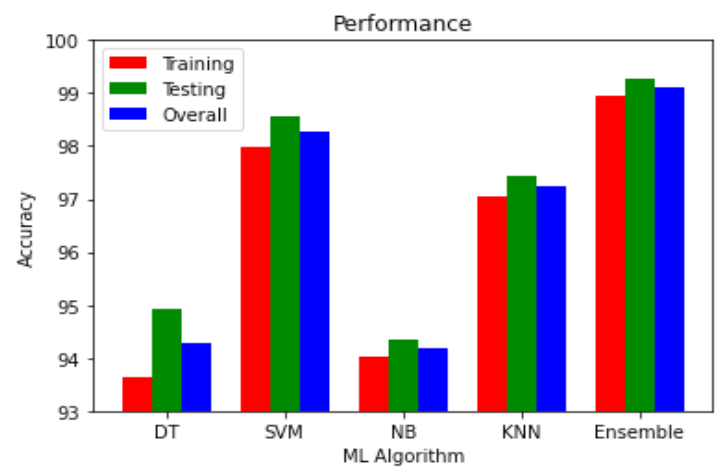

Figure 5. Prediction Accuracy Comparisons

This disparity can be attributed to the lower percentage of instances of $20 \%$ that are used during testing compared to $80 \%$ instances during training. To validate the proposed ensemble classification model, 10 folds cross validation was executed. To achieve this, the entire dataset is portioned in 10 equal sets. Next, 9 of these sets are deployed for training while the remaining 1 set is utilized for testing the model. This process is repeated ten times with each of the ten sub-samples being used at least once. The results obtained are depicted in Figure 6 below.

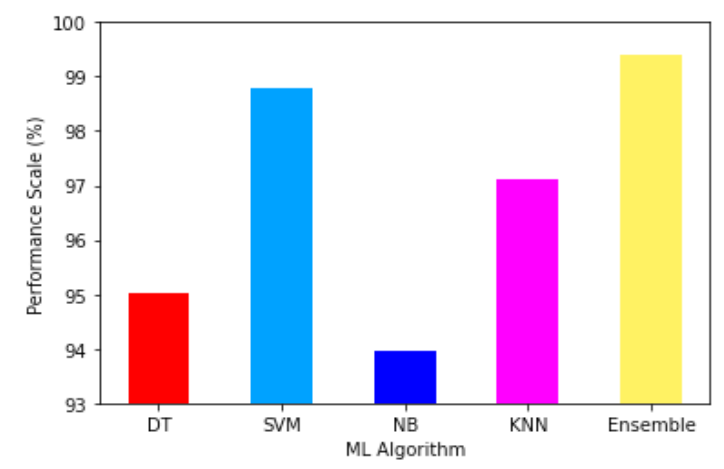

Figure 6. Cross Validation

It is evident from Figure 6 that NB classifier had the least performance of $93.96 \%$, followed by DT, KNN, SVM and the proposed ensemble classifier with $95.02 \%$, $97.11 \%, 98.79$ and $99.39 \%$ respectively. Based on the results above, it is evident that the deployed NCA feature selection and majority voting in the proposed classifier boosted precision, recall, F-measure and classification accuracy. This explains the slightly better performance of the proposed ensemble classifier when compared with individual classifiers.

\section{Conclusions}

Many machine learning algorithms have been developed to aid in optimization, prediction and diagnostics. However, these machine learning algorithms have been noted to have numerous challenges that may impede their effectiveness. These challenges revolve around the feature selection methods, imbalanced datasets and inability of learning generalization, among other issues. For instance, ensemble models perform better than individual classifiers, but the ideal selection of diversity classifier members to construct an ensemble, and the fusion of individual decisions of the base classifiers into a single decision present some setbacks. On their part, deep learning models have issues with the deployed weighting schemes. Moreover, the fat-short property of transcriptbased data reduces the prediction accuracies of some of the deep learning models. The developed ensemble classifier deploys a more effective feature selection technique to eliminate irrelevant features from the dataset. The results show that this ensemble classifier is more effective when compared to individual classifiers such as NB, KNN, DT and SVM. Future work lies in the testing 
of the developed classifier in different datasets to validate its effectiveness.

\section{References}

[1] Gupta, M., Gupta, B., 2018. A comparative study of breast cancer diagnosis using supervised machine learning techniques. in 2018 second international conference on computing methodologies and communication (ICCMC), IEEE. 997-1002.

[2] Alkeshuosh, A.H., Moghadam, M.Z., Al Mansoori, I., Abdar, M., 2017. Using PSO Algorithm for Producing Best Rules in Diagnosis of Heart Disease. in 2017 International Conference on Computer and Applications (ICCA), IEEE. 306-311.

[3] Nyangaresi, V.O., Rodrigues, A.J., Abeka, S.O., 2020. Secure Handover Protocol for High Speed 5G Networks. Int. J. Advanced Networking and Applications. 11(06), 4429-4442.

[4] Rashad, T., Sudhir, A., 2019. Fuzzy-Neural based Cost Effective Handover Prediction Technique for $5 \mathrm{G}-\mathrm{IoT}$ networks. International Journal of Innovative Technology and Exploring Engineering. 9(2S3), 191197.

[5] Mahira, A.G., Subhedar, M.S., 2017. Handover decision in wireless heterogeneous networks based on feed forward artificial neural network. in Computational Intelligence in Data Mining, Springer, Singapore. 663-669.

[6] Nyangaresi, V.O., Abeka, S.O., Rodgrigues, A., 2018. Secure Timing Advance Based Context-Aware Handover Protocol for Vehicular Ad-Hoc Heterogeneous Networks. International Journal of Cyber-Security and Digital Forensics. 7(3), 256-275.

[7] Wafa, B., Adnane, L., Vicent, P., 2019. Applying ANFIS Model in Decision-making of Vertical Handover between Macrocell and Femto-cell Integrated Network. Journal of Telecommunication, Electronic and Computer Engineering. 11(1), 57-62.

[8] Azzali, F., Ghazali, O., Omar, M.H., 2017. Fuzzy Logic-based Intelligent Scheme for Enhancing QoS of Vertical Handover Decision in Vehicular Ad-hoc Networks. International Research and Innovation Summit (IRIS2017). 226, 1-12.

[9] Nyangaresi, V.O., Abeka, S.O., Rodrigues, A.J., 2020. Delay Sensitive Protocol for High Availability LTE Handovers. American Journal of Networks and Communications. 9(1), 1-10.

[10] Shanmugam, K., 2017. A novel candidate network selection based handover management with fuzzy logic in heterogeneous wireless networks. in 4th International Conference on Advanced Computing and
Communication Systems (ICACCS), IEEE. 1-6.

[11] Aibinu, A., Onumanyi, J.A., Adedigba, P., Ipinyomi, M., Folorunso, T., Salami, M., 2017. Development of hybrid artificial intelligent based handover decision algorithm. Int. J. Eng. Sci. Technol. 20(2), 381-390.

[12] Nyangaresi, V.O., Rodrigues, A.J., Abeka, S.O., 2020. ANN-FL secure handover protocol for $5 \mathrm{G}$ and beyond networks. in International Conference on e-Infrastructure and e-Services for Developing Countries, Springer, Cham. 99-118.

[13] Zineb, A., Ayadi, M., Tabbane, S., 2017. QoE-based vertical handover decision management for cognitive networks using ANN. in Proceedings of the 2017 Sixth International Conference on Communications and Networking (ComNet), IEEE. 1-7.

[14] Eman, Z., Amr, A., Abdelkerim, T., Abdelhalim, Z., 2018. A novel vertical handover algorithm based on Adaptive Neuro-Fuzzy Inference System (ANFIS). International Journal of Engineering \& Technology. 7(1), 74-78.

[15] Nyangaresi, V.O., Rodrigues, A.J., 2022. Efficient handover protocol for $5 \mathrm{G}$ and beyond networks. Computers \& Security. 113, 102546.

[16] Pragati, K., Haridas, S.L., 2019. Reducing Ping-Pong Effect in Heterogeneous Wireless Networks Using Machine Learning. Intelligent Communication, Control and Devices. 697-705.

[17] Jamal, F.A., Firudin, K.M., 2017. Direction prediction assisted handover using the multilayer perception neural network to reduce the handover time delays in LTE networks. in 9th International Conference on Theory and Application of Soft Computing, Computing with Words and Perception, Procedia Computer Science. 120, 719-727.

[18] Nyangaresi, V.O., Rodrigues, A.J., Abeka, S.O., 2020. Neuro-Fuzzy Based Handover Authentication Protocol for Ultra Dense 5G Networks. in 2020 2nd Global Power, Energy and Communication Conference (GPECOM), IEEE. 339-344.

[19] Eshtay, M., Faris, H., Obeid, N., 2018. Improving extreme learning machine by competitive swarm optimization and its application for medical diagnosis problems. Expert Syst. Appl. 104, 134-152.

[20] Rosellini, A.J., Liu, S., Anderson, G.N., Sbi, S., Tung, E.S., Knyazhanskaya, E., 2020. Developing algorithms to predict adult onset internalizing disorders: An ensemble learning approach. Journal of psychiatric research. 121, 189-196.

[21] Sevakula, R.K., Verma, N.K., 2017. Assessing generalization ability of majority vote point classifiers. IEEE Transactions on Neural Networks and Learning 
Systems. 28(12), 2985-97.

[22] Miao, K.H., Miao, J.H., Miao, G.J., 2016. Diagnosing coronary heart disease using ensemble machine learning. Int J Adv Comput Sci Appl (IJACSA). $7(10), 1-12$.

[23] Li, H., Cui, Y., Liu, Y., Li, W., Shi, Y., Fang, C., Lu, Y., 2018. Ensemble learning for overall power conversion efficiency of the all-organic dye-sensitized solar cells. IEEE Access. 6, 34118-34126.

[24] An, N., Ding, H., Yang, J., Au, R., Ang, T.F., 2020. Deep ensemble learning for Alzheimer's disease classification. Journal of biomedical informatics. 105, 103411.

[25] Sun, J., Lang, J., Fujita, H., Li, H., 2018. Imbalanced enterprise credit evaluation with DTE-SBD: Decision tree ensemble based on SMOTE and bagging with differentiated sampling rates. Inf. Sci. 425,76-91.

[26] Zhang, X., Mahadevan, S., 2019. Ensemble machine learning models for aviation incident risk prediction. Decis. Support Syst. 116, 48-63.

[27] Mittal, D., Gaurav, D., Roy, S.S., 2015. An effective hybridized classifier for breast cancer diagnosis. in 2015 IEEE international conference on advanced intelligent mechatronics (AIM), IEEE,1026-1031.

[28] Jiang, J., Li, X., Zhao, C., Guan, Y., Yu, Q., 2017. Learning and inference in knowledge-based probabilistic model for medical diagnosis. Knowledge-Based Systems. 138, 58-68.

[29] Baccouche, A., Garcia-Zapirain, B., Castillo Olea, C., Elmaghraby, A., 2020. Ensemble Deep Learning Models for Heart Disease Classification: A Case Study from Mexico. Information. 11(4), 207.

[30] Wang, L., Zhou, W., Chang, Q., Chen, J., Zhou, X., 2019. Deep Ensemble Detection of Congestive Heart Failure using Short-term RR Intervals. IEEE Access. 7, 69559-69574.

[31] Liu, N., Li, X., Qi, E., Xu, M., Li, L., Gao, B., 2020. A novel Ensemble Learning Paradigm for Medical Diagnosis with Imbalanced Data. IEEE Access. 8, 171263-171280.

[32] Abdar, M., Zomorodi-Moghadam, M., Zhou, X., Gururajan, R., Tao, X., Barua, P.D., Gururajan, R., 2020. A new nested ensemble technique for automated diagnosis of breast cancer. Pattern Recognit. Lett. 132, 123-131.

[33] Kazemi, Y., Mirroshandel, S.A., 2018. A novel method for predicting kidney stone type using ensemble learning. Artif. Intell. Med. 84, 117-126.

[34] Wang, Y., Wang, D., Ye, X., Wang, Y., Yin, Y., Jin, Y., 2019. A tree ensemble based two-stage model for advanced-stage colorectal cancer survival prediction.
Inf. Sci. 474, 106-124.

[35] Nilashi, M., Ahmadi, H., Shahmoradi, L., Ibrahim, O., Akbari, E., 2019. A predictive method for hepatitis disease diagnosis using ensembles of neuro-fuzzy technique. Journal of infection and public health. 12(1), 13-20.

[36] Ali, F., El-Sappagh, S., Islam, S.R., Kwak, D., Ali, A., Imran, M., Kwak, K.S., 2020. A smart healthcare monitoring system for heart disease prediction based on ensemble deep learning and feature fusion. Information Fusion. 63, 208-222.

[37] Reddy, G.T., Bhattacharya, S., Ramakrishnan, S.S., Chowdhary, C.L., Hakak, S., Kaluri, R., Reddy, M.P.K., 2020. An ensemble based machine learning model for diabetic retinopathy classification. in 2020 international conference on emerging trends in information technology and engineering (ic-ETITE), IEEE. 1-6.

[38] Zhang, B., Qi, S., Monkam, P., Li, C., Yang, F., Yao, Y.D., Qian, W., 2019. Ensemble learners of multiple deep CNNs for pulmonary nodules classification using CT images. IEEE Access. 7, 110358-110371.

[39] Han, L., Luo, S., Yu, J., Pan, L., Chen, S., 2015. Rule extraction from support vector machines using ensemble learning approach: an application for diagnosis of diabetes. IEEE Journal of Biomedical and Health Informatics. 19(2), 728-34.

[40] Lindsey, T., Lee, J.J., 2020. Automated Cardiovascular Pathology Assessment Using Semantic Segmentation and Ensemble Learning. Journal of digital imaging. 1-6.

[41] Aličković, E., Subasi, A., 2017. Breast cancer diagnosis using GA feature selection and Rotation Forest. Neural Computing and Applications. 28(4), 753-763.

[42] Esfahani, H.A., Ghazanfari, M., 2017. Cardiovascular disease detection using a new ensemble classifier. in Proceedings of the 2017 IEEE 4th International Conference on Knowledge-Based Engineering and Innovation (KBEI), Tehran, Iran. 1011-1014.

[43] Yu, Y., Lin, H., Meng, J., Wei, X., Zhao, Z., 2017. Assembling deep neural networks for medical compound figure detection. Information. 8, 48.

[44] Pławiak, P., Acharya, U.R., 2020. Novel deep genetic ensemble of classifiers for arrhythmia detection using ECG signals. Neural Computing and Applications. 32(15), 11137-11161.

[45] Yekkala, I., Dixit, S., Jabbar, M.A., 2017. Prediction of heart disease using ensemble learning and Particle Swarm Optimization. in 2017 International Conference On Smart Technologies For Smart Nation (SmartTechCon), IEEE. 691-698. 
[46] Krishnaiah, V., Srinivas, M., Narsimha, G., Chandra, N.S., 2014. Diagnosis of heart disease patients using fuzzy classification technique. in International Conference on Computing and Communication Technologies, IEEE. 1-7.

[47] Moitra, D., Mandal, R.K., 2019. Automated AJCC staging of non-small cell lung cancer (NSCLC) using deep convolutional neural network (CNN) and recurrent neural network (RNN). Health information science and systems. 7(1), 1-12.

[48] Islam, M.M., Islam, M.Z., Asraf, A., Ding, W., 2021. Diagnosis of COVID-19 from X-rays using combined CNN-RNN architecture with transfer learning. MedRxiv. 2020-08.

[49] Sethy, P.K., Behera, S.K., Ratha, P.K., Biswas, P., 2020. Detection of Coronavirus Disease (COVID-19) based on Deep Features and Support Vector Machine. International Journal of Mathematical Engineering and Management Sciences. 643-651.

[50] Narin, A., Kaya, C., Pamuk, Z., 2021. Automatic detection of coronavirus disease (covid-19) using $\mathrm{X}$-ray images and deep convolutional neural networks. Pattern Analysis and Applications. 1-14.

[51] Loey, M., Smarandache, F., M Khalifa, N.E., 2020. Within the lack of chest COVID-19 X-ray dataset: a novel detection model based on GAN and deep transfer learning. Symmetry. 12(4), 651.

[52] Hemdan, E.E.D., Shouman, M.A., Karar, M.E., 2020. COVIDX-Net: A Framework of Deep Learning Classifiers to Diagnose COVID-19 in X-Ray Images, arXiv. 2003, 11055.

[53] Mienye, I.D., Sun, Y., Wang, Z., 2020. An improved ensemble learning approach for the prediction of heart disease risk. Informatics in Medicine Unlocked. 20, 100402.

[54] Tjahjadi, H., Ramli, K., 2020. Noninvasive Blood Pressure Classification Based on Photoplethysmography Using K-Nearest Neighbors Algorithm: A Feasibility Study. Information. 11, 93.

[55] Azizi, S., Bayat, S., Yan, P., Tahmasebi, A., Kwak, J.T., Xu, S., Abolmaesumi, P., 2018. Deep recurrent neural networks for prostate cancer detection: analysis of temporal enhanced ultrasound. IEEE transactions on medical imaging. 37(12), 2695-2703.

[56] Rossetto, A.M., Zhou, W., 2017. Deep learning for categorization of lung cancer CT images. in 2017 IEEE/ACM international conference on connected health: applications, systems and engineering technologies (CHASE), IEEE, Philadelphia, PA. 272273.

[57] Coudray, N., Ocampo, P.S., Sakellaropoulos, T., Narula, N., Snuderl, M., Fenyö, D., Moreira, A.L., Razavian, N., Tsirigos, A., 2018. Classification and mutation prediction from non-small cell lung cancer histopathology images using deep learning. Nature medicine. 24(10), 1559-1567.

[58] Punn, N.S., Agarwal, S., 2021. Automated diagnosis of COVID-19 with limited posteroanterior chest $\mathrm{X}$-ray images using fine-tuned deep neural networks. Applied Intelligence. 51(5), 2689-2702.

[59] Khan, M.A., 2020. An IoT Framework for Heart Disease Prediction Based on MDCNN Classifier. IEEE Access. 8, 34717-34727. 